\title{
Nonlinear Soil Change by Exotic Earthworms: Organisms as a Soil Forming Factor
}

\author{
KYUNGSOO YOO $^{1 *}$, ADRIAN WACKETT ${ }^{1}$, TYLER \\ BAUMANN $^{1}$, LEE FRELICH ${ }^{1}$, DEREK SIKES ${ }^{2}$, MATT \\ BOWSER $^{3}$, STEPHEN BROWN ${ }^{2}$, CLAUDIA IHL ${ }^{2}$, MINGCHU \\ ZHANG $^{2}$, JULIE RILEY ${ }^{2}$, JONATAN KLAMINDER ${ }^{4}$
}

${ }^{1}$ Univ. of Minnesota, *corresponding: kyoo@umn.edu

${ }^{2}$ Univ. of Alaska, Fairbanks.

${ }^{3}$ Kenai National Wildlife Refuge

${ }^{4}$ Umeå Univ.

Understanding the coevolution of organisms and lands is one of the primary goals of biogeochemistry. However, studying organisms as a soil-forming factor is challenging. Population density, composition, and behavior of organisms respond to other soil-forming factors.

Here we present biosequences: soils differ solely because of the presence and absence of exotic earthworms. These biosequences are from temperate forests, boreal forests, and tundra biomes that have evolved with little or no faunal pedoturbation. The last glaciation eradicated native earthworms, but such status quo was recently reset by humans who served as a vector of European earthworms.

We report on a number of earthworm invasion biosequences across formerly glaciated biomes in Minnesota, Fennoscandia, and Alaska. We observed: (1) Human cultures affect the combination of introduced earthworm species and thus set the first-order control on available earthworm populations in a given location. (2) Among those introduced, only the earthworm species that are compatible with the climate and existing soil environments further disperse into natural vegetation. (3) Where geoengineering earthworms are introduced, survive, and disperse, soils are subject to rapid non-linear changes in morphology, elemental mass balances, and the ways and extents that organic matter and minerals interact.

Invasive geoengineering earthworms push the soils across the threshold from mors to mulls within a decade. Such nonlinear soil shift by invasive earthworms is yet scattered but not uncommon in the arctic Sweden and Alaska. As the warming arctic lures human activities, increasingly larger areas will be invaded by European geoengineering earthworms and subsequently undergo the shift from mors to mulls. This biosequence study warns that the emerging faunal pedoturbation may turn out to be a smoking gun for the fate of the soils and organic matter on the earth's high latitude biomes. 